\title{
Duality in Solving Multi-Objective Optimization (M00) Problems
}

\author{
Chandra Sen \\ Department of Agricultural Economics, Institute of Agricultural Sciences, Banaras Hindu University, Varanasi, India \\ Email: chandra_sen@rediffmail.com
}

How to cite this paper: Sen, C. (2019) Duality in Solving Multi-Objective Optimization (MOO) Problems. American Journal of Operations Research, 9, 109-113. https://doi.org/10.4236/ajor.2019.93006

Received: April 20, 2019

Accepted: May 18, 2019

Published: May 21, 2019

Copyright (c) 2019 by author(s) and Scientific Research Publishing Inc. This work is licensed under the Creative Commons Attribution International License (CC BY 4.0).

http://creativecommons.org/licenses/by/4.0/

\begin{abstract}
Multi-Objective Optimization (MOO) techniques often achieve the combination of both maximization and minimization objectives. The study suggests scalarizing the multi-objective functions simpler using duality. An example of four objective functions has been solved using duality with satisfactory results.

\section{Keywords}

Duality, Multi-Objective Optimization (MOO), Scalarizing Techniques
\end{abstract}

\section{Introduction}

Multi-Objective Optimization helps in making decisions in presence of usually conflicting objectives. Scalarizing techniques have been popularly used for solving multi-objective optimization problems. Several new scalarizing techniques [1]-[11] have been proposed during recent years. These scalarizing techniques are not efficient [12] [13] in optimizing the multiple objectives simultaneously. An improved scalarizing technique is proposed for solving MOO problems. Duality can be used to formulate the multi-objective function easier. The present study explains the utility of duality in solving multi-objective optimization problem with a suitable example.

\section{Sen's Multi-Objective Optimization Technique}

\subsection{Primal Multi-Objective Function}

The mathematical form of Sen's MOO technique [12] [13] is described as:

Optimize $Z=\left[\right.$ Max. $Z_{1}$, Max. $Z_{2}, \cdots$, Max. $Z_{r}$, Min. $Z_{r+1}, \cdots$, Min. $\left.Z_{s}\right]$

Subject to:

$$
A X=b \text { and } X \geq 0
$$


The individual optima are obtained by optimizing each objective separately as:

$$
Z_{\text {optima }}=\left[\theta_{1}, \theta_{2}, \cdots, \theta_{s}\right]
$$

The Primal Multi-Objective Function is formulated as:

Maximize $Z=\frac{\sum_{j=1}^{r} Z_{j}}{\left|\theta_{j}\right|}-\frac{\sum_{j=r+1}^{s} Z_{j}}{\left|\theta_{r+1}\right|}$

Subject to:

$$
\begin{gathered}
A X=b \text { and } X \geq 0 \\
\theta_{j} \neq 0 \text { for } j=1,2, \cdots, s .
\end{gathered}
$$

where, $\theta_{j}$ is the optimal value of $j$ th objective function.

\subsection{Dual Multi-Objective Function}

All the objective functions are converted into either maximizing or minimizing form as described below:

Maximize $Z_{j}$ or Minimize $Z_{j}$

Subject to:

$$
A X=b \text { and } X \geq 0
$$

The minimization objective function can be converted into maximization objective function by multiplying -1 . Similarly the maximization objective can be converted into minimization objective function by multiplying -1 . The Multi-Objective Function is formulated as:

Maximize $Z=\frac{\sum_{j=1}^{s} Z_{j}}{\left|\theta_{j}\right|}$

or

Minimize $Z=\frac{\sum_{j=1}^{s} Z_{j}}{\left|\theta_{j}\right|}$

Subject to:

$$
\begin{gathered}
A X=b \text { and } X \geq 0 \\
\theta_{j} \neq 0 \text { for } j=1,2, \cdots, s .
\end{gathered}
$$

where, $\theta_{j}$ is the optimal value of $j$ th objective function.

\section{Algorithm of Proposed Technique}

Step I: Convert all the objective functions either maximization of minimization mode.

Step II: Formulate multi-objective function as explained in 2.2

Step III: Optimize the multi-objective function under the same constraints.

\section{Multi-Objective Optimization Problem}

The following example has been solved with duality technique.

Example 
$\operatorname{Max} . Z_{1}=12500 X_{1}+25100 X_{2}+16700 X_{3}+23300 X_{4}+20200 X_{5}$

$\operatorname{Max} . Z_{2}=21 X_{1}+15 X_{2}+13 X_{3}+17 X_{4}+11 X_{5}$

Min. $Z_{3}=370 X_{1}+280 X_{2}+350 X_{3}+270 X_{4}+240 X_{5}$

Min. $Z_{4}=1930 X_{1}+1790 X_{2}+1520 X_{3}+1690 X_{4}+1720 X_{5}$

Subject to:

$$
\begin{gathered}
X_{1}+X_{2}+X_{3}+X_{4}+X_{5}=4.5 \\
2 X_{1} \geq 1.0 \\
3 X_{4} \geq 1.5
\end{gathered}
$$

The above problem can be converted with all the four objective functions either maximization of minimization mode as detailed below:

$$
\begin{aligned}
& \text { Max. } Z_{1}=12500 X_{1}+25100 X_{2}+16700 X_{3}+23300 X_{4}+20200 X_{5} \\
& \text { Max. } Z_{2}=21 X_{1}+15 X_{2}+13 X_{3}+17 X_{4}+11 X_{5} \\
& \text { Max. } Z_{3}=-370 X_{1}-280 X_{2}-350 X_{3}-270 X_{4}-240 X_{5} \\
& \text { Max. } Z_{4}=-1930 X_{1}-1790 X_{2}-1520 X_{3}-1690 X_{4}-1720 X_{5}
\end{aligned}
$$

or

$$
\begin{aligned}
& \text { Min. } Z_{1}=-12500 X_{1}-25100 X_{2}-16700 X_{3}-23300 X_{4}-20200 X_{5} \\
& \text { Min. } Z_{2}=-21 X_{1}-15 X_{2}-13 X_{3}-17 X_{4}-11 X_{5} \\
& \text { Min. } Z_{3}=370 X_{1}+280 X_{2}+350 X_{3}+270 X_{4}+240 X_{5} \\
& \text { Min. } Z_{4}=1930 X_{1}+1790 X_{2}+1520 X_{3}+1690 X_{4}+1720 X_{5}
\end{aligned}
$$

The problem was solved with multi-objective function of both maximization and minimization mode. It is very clear from Table 1 that all the four individual optimizations are all different and do not achieve all the objectives simultaneously.

This necessitates the need of multi-objective optimization. Both the solutions of multi-objective optimization are exactly the same and achieving all the four objectives simultaneously. Hence the multi-objective optimization problems can be solved by formulating multi-objective function after converting all the objective functions in either maximizing or minimizing mode.

Table 1. Individual and multi-objective optimization.

\begin{tabular}{ccccccc}
\hline \multirow{2}{*}{$\begin{array}{c}\text { Objective } \\
\text { Function }\end{array}$} & \multicolumn{3}{c}{ Individual Optimization } & & \multicolumn{2}{c}{ Multi-Objective Optimization } \\
\cline { 3 - 7 } & Max. $Z_{1}$ & Max. $Z_{2}$ & Min. $Z_{3}$ & Min. $Z_{4}$ & $\begin{array}{c}\text { Maximization } \\
\text { Mode }\end{array}$ & $\begin{array}{c}\text { Minimization } \\
\text { Mode }\end{array}$ \\
\hline \multirow{2}{*}{$X_{i}$} & $0.5,3.5$, & $4,0,0$, & $0.5,0,0$, & $0.5,0,3.5$, & $0.5,0,0,4,0$ & $0.5,0,0,4,0$ \\
& $0,0.5,0$ & $0.5,0$ & $0.5,3.5$ & $0.5,0$ & & \\
$Z_{1}$ & 105750 & 61650 & 88600 & 76350 & 99450 & 99450 \\
$Z_{2}$ & 71.5 & 92.5 & 57.5 & 64.5 & 78.5 & 78.5 \\
$Z_{3}$ & 1300 & 1615 & 1160 & 1545 & 1265 & 1265 \\
$Z_{4}$ & 8075 & 8565 & 7830 & 7130 & 7725 & 7725 \\
\hline
\end{tabular}




\section{Conclusion}

One of the important advantages of the duality theory is presented in the paper for solving MOO problems. It is established that duality makes easier the formulation of multi-objective function. However, it is needed only when optimization is done for a set of both maximization and minimization objective functions.

\section{Conflicts of Interest}

The author declares no conflicts of interest regarding the publication of this paper.

\section{References}

[1] Sulaiman, N.A. and Hamadameen, A.-Q.O. (2008) Optimal Transformation Technique to Solve Multi-Objective Linear Programming Problem (MOLPP). Journal of Kirkuk University Scientific Studies, 3, 158-168.

[2] Suleiman, N.A. and Nawkhass, M.A. (2013) Transforming and Solving Multi-Objective Quadratic Fractional Programming Problems by Optimal Average of Maximin \& Minimax Techniques. American Journal of Operational Research, 3, 92-98.

[3] Sulaiman, N.A. and Mustafa, R.B. (2016) Using Harmonic Mean to Solve Multi-Objective Linear Programming Problems. American Journal of Operations Research, 6, 25-30. https://doi.org/10.4236/ajor.2016.61004

[4] Sulaiman, N.A. and Mustafa, R.B. (2016) Transform Extreme Point Multi-Objective Linear Programming Problem to Extreme Point Single Objective Linear Programming Problem by Using Harmonic Mean. Applied Mathematics, 6, 95-99.

[5] Huma, A., Geeta, M. and Sushma, D. (2017) Transforming and Optimizing Multi-Objective Quadratic Fractional Programming Problem. International Journal of Statistics and Applied Mathematics, 2, 01-05.

[6] Nahar, S. and Alim, A. (2017) A New Statistical Averaging Method to Solve Multi-Objective Linear Programming Problem. International Journal of Science and Research, 6, 623-629.

[7] Huma, A., Modi, G. and Duraphe, S. (2017) An Appropriate Approach for Transforming and Optimizing Multi-Objective Quadratic Fractional Programming Problem. International Journal of Mathematics Trends and Technology, 50, 80-83. https://doi.org/10.14445/22315373/IJMTT-V50P511

[8] Nawkhass, M.A. and Birdawod, H.Q. (2017) Transformed and Solving Multi-Objective Linear Programming Problems to Single-Objective by Using Correlation Technique. Cihan International Journal of Social Science, 1, 30-36.

[9] Akhtar, H. and Modi, G. (2017) An Approach for Solving Multi-Objective Fractional Programming Problem and It's Comparison with Other Techniques. International Journal of Scientific and Innovative Mathematical Research, 5, 1-5. https://doi.org/10.20431/2347-3142.0511001

[10] Nahar, S. and Alim, A. (2017) A New Geometric Average Technique to Solve Multi-Objective Linear Fractional Programming Problem and Comparison with New Arithmetic Average Technique. IOSR Journal of Mathematics (IOSR-JM), 13, 39-52. https://doi.org/10.9790/5728-1303013952

[11] Sohag, Z.I. and Asadujjaman, Md. (2018) A Proposed New Average Method for 
Solving Multi-Objective Linear Programming Problem Using Various Kinds of Mean Techniques. Mathematics Letters, 4, 25-33.

https://doi.org/10.11648/j.ml.20180402.11

[12] Sen, C. (2018) Multi Objective Optimization Techniques: Misconceptions and Clarifications. International Journal of Scientific and Innovative Mathematical Research, 6, 29-33.

[13] Sen, C. (2018) Sen's Multi-Objective Programming Method and Its Comparison with Other Techniques. American Journal of Operational Research, 8, 10-13. 